\title{
APRV Mode in Ventilator Induced Lung Injury (VILI)
}

Ata Mahmoodpoor ${ }^{1}$, Samad EJ Golzari ${ }^{2 *}$

1. Cardiovascular Research Center, Tabriz University of Medical Science, Tabriz, Iran

2. Medical Philosophy and History Research Center, Tabriz University of Medical Sciences, Tabriz, Iran

\section{Editorial}

Ventilator-Induced Lung Injury (VILI), being a significant iatrogenic complication in the ICU patients, is associated with high morbidity and mortality. Numerous approaches, protocols and ventilation modes have been introduced and examined to decrease the incidence of VILI in the ICU patients. Airway pressure release ventilation (APRV), firstly introduced by Stock and Downs in 1987, applies higher Continuous Positive Airway Pressure (CPAP) levels in prolonged periods ( $\mathrm{P}$ and $\mathrm{T}$ high) in order to preserve satisfactory lung volume and consequently alveolar recruitment. This mode benefits a time-cycled release phase to a lower set of pressure for a short period of time ( $P$ and $T$ low i.e. release time) $(1,2)$. While some advantages have been introduced for APRV such as efficiently recruited alveoli over time, more homogeneous ventilation, less volutrauma, probable stabilization of patent alveoli and reduction in atelectrauma, protective effects of APRV on lung damage only seem to be substantial if spontaneous breathing responds to more than $30 \%$ of total minute ventilation (3). APRV in ARDS patients should be administered cautiously; $T$ low $<0.6$ seconds, for recruiting collapsed alveoli; however overstretching of alveoli especially during $P$ high should not be neglected and appropriate sedation considered.

The proposed advantages for APRV give the impression of being outstanding; however, APRV, as a non-physiologic inverse ratio mode of ventilation, might result in inflammation mainly due to impaired patient-ventilator interaction explaining the negative or minimally desirable effects of APRV on inflammation (4). Consequently, continuous infusion of neuromuscular blocking drugs during ARDS has been reported to reduce mortality (5). There are insufficient confirming data on the superiority of APRV above other ventilatory methods in regard to oxygenation, hemodynamics, regional blood flow, patient comfort and length of mechanical ventilation. Based on current findings and the concept that ARDS is not a syndrome that should be treated, but rather prevented, APRV does not seem to be an ideal mode for prevention of ARDS in mechanically ventilated subjects especially in those with concurrent intra-cranial hypertension and large broncho-pleural fistulae (5). If APRV is opted for ARDS patients, it is

\section{Corresponding author:}

Samad EJ Golzari

Cardiovascular Research Center, Tabriz University of Medical Science, Tabriz, Iran

Tel/Fax: +989141151894 E-mail: dr.golzari@hotmail.com

Received: 15-07-2013 Accepted: 16-07-2013 Published: 07-01-2014

doi:10.7575/aiac.abcmed.v.2n.1p.1 
recommended to use appropriate sedation and $\mathrm{T}$ low 0.6 seconds for recruiting collapsed alveoli and be cautious regarding overstretching of alveoli especially during $P$ high. Currently APRV is recommended for carefully-selected and not all ALI/ ARDS patient.

\section{References}

1. Downs JB, Stock MC. Airway pressure release ventilation: A new concept in ventilatory support. Crit Care Med. 1987;15:459-61.

2. Stock MC, Downs JB, Frolicher DA. Airway pressure release ventilation. Crit Care Med. 1987;15:462-6.

3. Habashi NM. Other approaches to open-lung ventilation: Airway pressure release ventilation. Crit Care Med. 2005;33:S228-40.

4- Gama de Abreu M, Spieth PM, Pelosi P, et al. Noisy pressure support ventilation: a pilot study on a new assisted ventilation mode in experimental lung injury. Crit Care Med 2008; 36: 818-827.

5- Papazian L, Forel JM, Gacouin A, et al. Neuromuscular blockers in early acute respiratory distress syndrome. N Engl J Med 2010; 363: 1107-1116. 